[Agr. Biol. Chem., Vol. 34, No. 4, p. 568 574, 1970]

\title{
Complexes of Starchy Materials with Organic Compounds
}

\author{
Part IV X-Ray Diffraction of $\gamma$-Cyclodextrin Complexes ${ }^{\dagger}$ \\ By Ken'ichi Takeo and Takashi Kuge \\ Department of Agricultural Chemistry, Kyoto Prefectural University, Kyoto \\ Received August 27, 1969
}

\begin{abstract}
The crystal structure of $\gamma$-cyclodextrin complexes with several organic compounds have been investigated by $\mathrm{X}$-ray powder method. A two-dimensional tetragonal unit cell having $\mathrm{a}=\mathrm{b}=27.2 \AA$ and a two-dimensional hexagonal unit cell having $\mathrm{a}=\mathrm{b}=32.7 \AA$, were reasonably proposed for hydrated and anhydrous $\gamma$-dextrin complexes, respectively. The change of the crystal structure caused by dehydration seemed to be resulted from the change of the packing arrangement of circular cylinders that are made by coaxial alignment of the dextrin molecules. Results obtained were tentatively applied to consider the $8_{1}$-helical configuration of amylose.
\end{abstract}

In the preceding paper, ${ }^{11}$ it has been demonstrated that the crystal structures of amylose complexes with a number of organic compounds are very reasonably established by comparison of their X-ray diffraction diagrams with those of cyclodextrin complexes. It was shown that the amylose complexes in thoroughly dried state exist in two kinds of helical configurations, depending upon the types of the complexing agents used. The configurations were both interpreted in terms of a hexagonal unit cell, which can be accounted for by assuming a closest packing of helices containing six or seven glucose residues per helical turn $\left(6_{1}\right.$-helix or $7_{1}$-helix). It was also found that the mutual structural transitions between both helices were observed in the wet precipitate of the amylose complexes on the treatment of the complexes with suitable alcohols miscible with water.

The ease of the change of the helical structure of amylose complexes would now lead

\footnotetext{
$t$ Presented at the Annual Meeting of the Agricultural Chemical Society of Japan, Tokyo, April 1, 1969.

1) K. Takeo and T. Kuge, Agr. Biol. Chem., 33, 1174 (1969).
}

us to expect another possible helical structure, i.e., $8_{1}$-helix amylose, suggested by Senti et al.," which has a large cross section than those of

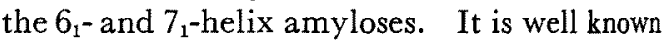
that $\gamma$-cyclodextrin with cyclic structure, which is composed of eight $\alpha$-D-glucopyranose units linked in $\alpha-1,4$ as in amylose, occurs in the products obtained by the Bacillus macerans enzymolysis of starch, besides $\alpha$ - and $\beta$ cyclodextrins. In the formation of the dextrins, it may be considered ${ }^{3}$ that the linear chain of starch would take a helical arrangement before the ring-closing reaction of the enzyme, so it seems likely that the 8 -helical loop as well as the $6_{1}$ - and $7_{1}$-helical loops may exist in the starch solution prior to the action of the enzyme.

However, the crystal structure of the $8_{1}$-helix amylose is little known at present. The only description concerned with the structure is that reported by Senti et al.,$^{21}$ who described, without experimental details, that a fiber

2) F. R. Senti and S. R. Erlander, "Non-stoichiometric compounds," ed. by L. Mandelcorn, Academic Press., New York, 1964, p. 567.

3) D. French, Advances in Carbohydrate Chemistry, 12, 189 (1957). 
pattern from moist tert-butyl alcohol amylose complex may be indexed in terms of a monoclinic unit cell parameters and this complex probably contains eight $\mathrm{D}$-glucose residues per helical turn.

As pointed out in the preceding paper, ${ }^{11} \mathrm{X}$ ray diffraction studies of $\alpha$ - and $\beta$-cyclodextrin complexes have provided valuable informations about the crystal structure of the $61^{-}$and $7_{1}$ helix amylose complexes and the similarity of the structures between amylose and dextrin complexes could be established by comparison of their diffraction patterns. From this point of view, it seems very important to clarify the crystal structure of $\gamma$-dextrin complexes, since the correlations between the diffraction data of the dextrin complexes and the $8_{1}$-helix amylose may be useful to explain the configuration of the $8_{1}$-helix amylose.

\section{MATERIALS AND METHOD}

Materials. Potato starch used was the commercial sample of "Nippon Yakkyoku-hô" (moisture content; $17.5 \%)$.

Reagents. The reagents used were commercially available and of the purest grade. They were used without further purification.

Preparation of $\gamma$-cyclodextrin. $\gamma$-Cyclodextrin was prepared from potato starch by the action of Bacillus macerans amylase. The basic procedure was essentially the same as that described by French et al.4 Bacillus macerans* was grown at $38^{\circ} \mathrm{C}$ for 2 weeks on a mushed potato which was autoclaved and buffered with calcium carbonate, and the solution of enzyme thus obtained was used without further purification. The $2 \%$ starch solution was autoclaved at $120^{\circ} \mathrm{C}$ for $30 \mathrm{~min}$. The resulting solution was adjusted to $\mathrm{pH} 5.7$ with acetic acid and incubated with the enzyme solution $(30$ units**/g starch) at $38^{\circ} \mathrm{C}$ for $120 \mathrm{hr}$ to permit the production of $\gamma$-dextrin as well as $\alpha$ - and $\beta$-dextrins.

* IFO NO 3490 supplied from Institute for Fermentation of Osaka.

** estimated according to the method described by Tilden et al.s

4) D. French, M. L. Levine, J. H. Pazur and E. Norberg, J. Am. Chem. Soc., 71, 353 (1949).
At the end of this period, $\gamma$-dextrin was detectable in the products of the enzymolysis. The solution was then boiled to make the enzyme inactive. $\gamma$-Dextrin was separated from other dextrins after treatments of several steps which will be presented elsewhere. Large crystals of $\gamma$-dextrin were finally obtained from an aqueous solution by recrystallization and dried in vacuo at $100^{\circ} \mathrm{C}$ over phosphorus pentoxide for $24 \mathrm{hr}$. It showed specific rotatory power of $[\alpha]_{\mathrm{D}}^{25}=+177.5^{\circ}$, being in good agreement with that given by French et al.4

Preparation of $\gamma$-cyclodextrin complexes. The method used for the preparation of $\gamma$-dextrin complexes was essentially the same as that described in the preceding paper. ${ }^{11} \gamma$-Dextrin- $n$-propyl alcohol complex was prepared as follows. The pure dextrin $(0.5 \mathrm{~g})$ was dissolved in deionized water $(3 \mathrm{ml})$, and $n$-propyl alcohol $(5 \mathrm{ml})$ was added dropwise to the solution. Care was taken to make sure slow cooling of the solution so that no immediate precipitation may occur. After standing for a few days, large crystals of the complex were formed. The crystals were collected on a filter paper by suction filtration and subsequently dried in the air. Dextrin complexes with benzene, bromobenzene, cyclohexane and $p$-cymene were prepared as follows. The dextrin $(0.5 \mathrm{~g})$ in deionized water $(10 \mathrm{ml})$ was stirred overnight with each of the reagents $(0.5 \mathrm{ml})$ at room temperature to insure the complete complex formation. The crystalline complexes were then precipitated and filtered off. They were dried in the air. Anthracene complex was prepared by shaking continuously $0.5 \%$ aqueous solution of the dextrin with ether saturated with anthracene for several days. After the upper ether layer was decanted with care not to cause the contamination due to anthracene, the complex was filtered off and dried in the air.

$X$-Ray diffraction measurement. X-Ray diffraction diagrams were recorded by a powder method using Shimadzu X-ray diffractometer GX. The operation conditions used was the same as that used in the previous paper.11

\section{RESULTS AND DISCUSSION}

As previously observed for the crystals of $\alpha$ - and $\beta$-cyclodextrins" also in the case of $\gamma$ -

5) E. B. Tilden and C.S. Hudson, J. Bact., 43, 527 (1942). 
cyclodextrin, several kinds of X-ray diffraction patterns were obtained depending upon the extent of hydration. Figure 1-a shows the diffraction diagram of $\gamma$-dextrin which is dried
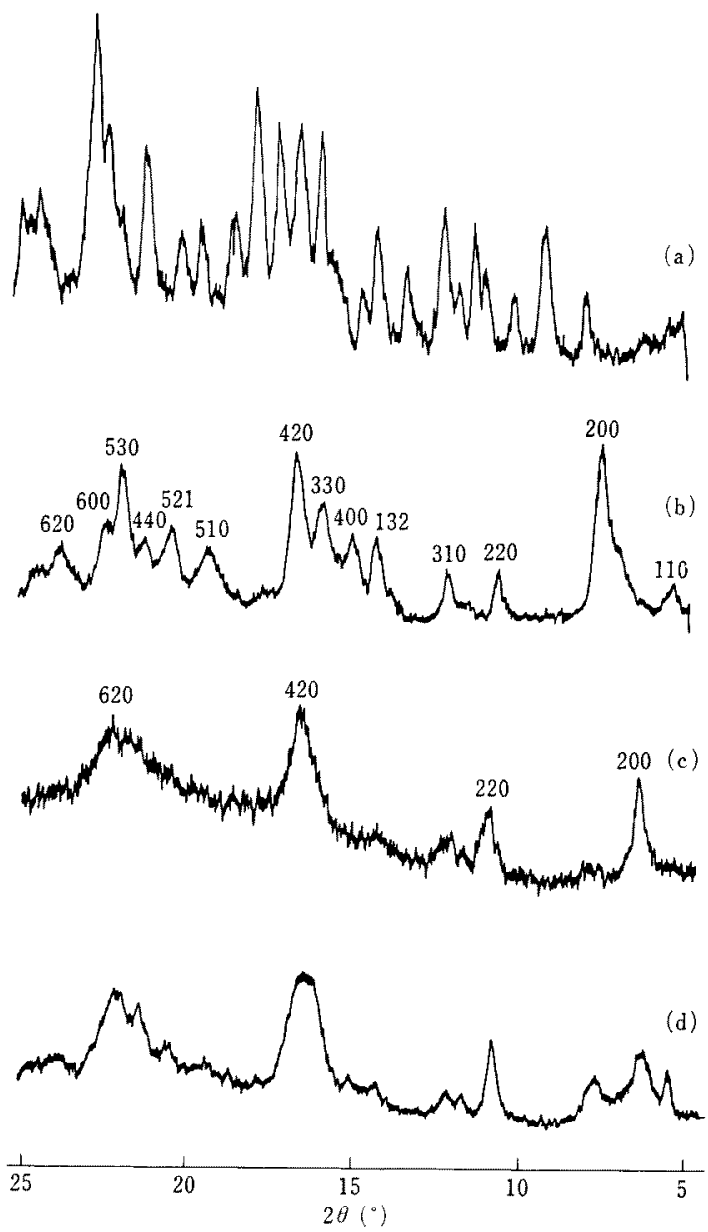

FIG. 1. X-Ray Diffraction Diagrams of the Powder Samples of $\gamma$-Cyclodextrin and its $n$-Propyl Alcohol Complex in Various Drying States.

a) Pure $\gamma$-cyclodextrin dried in the air.

b) $r$-Cyclodextrin- $n$-propyl alcohol complex dried in the air.

c) $\gamma$-Cyclodextrin-n-propyl alcohol complex dried in vacuo over phosphorus pentoxide at $100^{\circ} \mathrm{C}$ for $3 \mathrm{hr}$.

d) $\gamma$-Cyclodextrin-n-propyl alcohol complex dried in vacuo over silica gel at room temperature. in the air. It is impossible to determine unambiguously the crystal structure, since the pattern is too complex to be reliably indexed by the powder method alone due to many scattered lines observed. However, the dextrin complexes with organic compounds show rather simple patterns which enable us to determine the crystal structure. In the present study, $\gamma$-dextrin complexes with $n$-propyl alcohol, benzene, bromobenzene, cyclohexane, $p$-cymene and anthracene were prepared. For each complexes, X-ray diffraction diagrams were taken under various drying conditions.

When the dextrin complexes were dried in the air, each complex showed an identical diagram which can be characterized by the diffraction pattern of $\gamma$-dextrin- $n$-propyl alcohol complex as illustrated in Fig. 1-b, irrespective of the kinds of the complexing agents used ( $n$-propyl alcohol, benzene, cyclohexane, $p$ cymene, bromobenzene and anthracene). The diffraction pattern of $n$-propyl alcohol complex can be indexed on the basis of a tetragonal unit cell. The d-spacings of (200) line can be used to establish $a$ and $b$ axes of a two-

Table I. Diffraction Data For $\gamma$-Cyclodextrinn-PROPYL ALCOHOL COMPLEX

\begin{tabular}{crc}
\hline & \multicolumn{2}{c}{$\mathrm{d}, \AA$} \\
\cline { 2 - 3 } & Calcd. & Obs. \\
\hline 110 & 16.74 & 16.70 \\
200 & 11.85 & 11.85 \\
220 & 8.39 & 8.40 \\
310 & 7.50 & 7.45 \\
400 & 5.92 & 5.92 \\
330 & 5.59 & 5.60 \\
420 & 5.30 & 5.30 \\
510 & 4.65 & 4.65 \\
440 & 4.20 & 4.19 \\
530 & 4.06 & 4.06 \\
600 & 3.95 & 3.96 \\
620 & 3.75 & 3.75 \\
521 & 4.33 & 4.35 \\
132 & 6.27 & 6.24 \\
\hline
\end{tabular}

a) Tetragonal indices. 
dimensional unit cell which drawn in Fig. 2-a and all of the remaining equatorial reflection lines observed are reasonably indexed. Assigned indices for each reflection lines are indicated in the diagram. The calculated d-spacings from the two-dimensional tetragonal unit cell having $a=b=23.7 \AA$ agreed well with observed ones as seen in Table I. Hence, the packing diameter of circle drawn in Fig. 2-a was calculated to be $16.75 \AA$. The circle may correspond to the cross section of a cylinder that may be constructed from the coaxial alignment of the dextrin molecules (cf. Fig. 3-b). The value of the a axis was in good agreement with that given by French et al. ${ }^{6}$ The (521) and (132) lines were indexed by assuming that the unit length of $c$ axis is equal to $22.2 \mathrm{~A}$ as given by French et al. ${ }^{61}$ and by using auxiliary data obtained from Hull-Davey table. ${ }^{7}$

On the other hand, when the same specimen of $\gamma$-dextrin complex was dried over phosphorus pentoxide in vacuo at $100^{\circ} \mathrm{C}$ for $3 \mathrm{hr}$, the diffraction diagram as shown in Fig. 1-c was obtained. The pattern indicates that the crystal structure is different from that of specimen dried in the air. In this case, the diagram obviously exhibits the hexagonal nature of the pattern. In fact, the pattern can be interpreted by being indexed on the basis of a two-dimensional hexagonal unit cell having $a=b=32.7 \AA$, where the $d$-spacing for (200) line on powder diagram was used to calculate the unit cell dimensions. Assigned indices, observed and calculated d-spacings for the hexagonal unit cell are shown in Table II. The diameter of the cylinders was then calculated to be $16.36 \AA$ and the hexagonal unit cell was schematically shown in Fig. 2-b. Consequently, a decrease in the packing diameter by $0.39 \AA$ as compared with that of tetragonal unit cell occurs with drying of the complex.

When the specimen of the $n$-propyl alcohol

6) D. French, D. W. Knapp and J. H. Pazur, J. Am. Chem. Soc., 72, 5150 (1950).

7) A. W. Hull and W.P. Davey, Phys. Rev., 17, 549 (1921).
TABle II. DIFFraction DATA FOR $\gamma$-CYClODEXTRINn-PROPYL ALCOHOL COMPLEX

\begin{tabular}{crc}
\hline \multirow{2}{*}{ hkol $^{a} \mathrm{~d}, \AA$} \\
\cline { 2 - 3 } & Calcd. & Obs. \\
\hline 200 & 14.15 & 14.13 \\
220 & 8.18 & 8.18 \\
420 & 5.37 & 5.34 \\
620 & 3.94 & 4.00 \\
\hline
\end{tabular}

a) Hexagonal indices.

complex was allowed to dry in vacuo over silica gel at room temperature for $5 \mathrm{hr}$, the diffraction diagram as shown in Fig. 1-d was obtained. Neither the tetragonal unit cell nor the hexagonal one accounts for the diffraction pattern observed. However, when this specimen was subjected to an additional evacuation over phosphorus pentoxide at $80^{\circ} \mathrm{C}$ for $2 \mathrm{hr}$, the reflection lines of the powder diagram closely agreed with that of Fig. 1-c. Therefore, it seems probable that the diagram of Fig. 1-d shows an intermediate situation involving a transitional state from tetragonal unit cell to hexagonal one. All of the dextrin complexes investigated also exhibited the same change of the crystal structure in the course of the drying as do the $r$-dextrin- $n$-propyl alcohol complex. This results may suggest that the complexing agents included have little to do with the transition of the unit cell. The complexing agents seem to be strongly held within the void of the cyclodextrin ${ }^{4}$ and remain unchanged for the most part after the evacuation mentioned above. It seems, therefore, likely that the transition of the unit cell may take place with changing extent of hydration. The hydration would occur in interstices among cylinders formed by the dextrins, considering some analogy of the crystal with that of amylose-iodine complex. ${ }^{81}$ Consequently, it may be safely said that such

8) B. Zaslow and R. L. Miller, J. Am. Chem. Soc., 83, 4378 (1961). 
(a) Tetragonal unit cel! (Hydrated form)

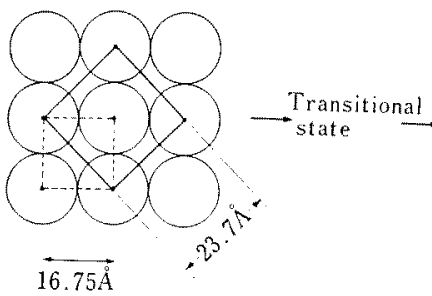

FIG. 2. Proposed Transition of the Packing Arrangements of the Cylinders in the $r$-Cyclodextrin Complexes.

a dehydration may cause the significant change on the crystal structure through the change of the packing arrangement of the dextrin cylinders. Hence, the tetragonal and hexagonal unit cells proposed for $\gamma$-dextrin complexes may corresponds to those of hydrated and anhydrous form, respectively. The transition of the unit cell for $\gamma$-dextrin complexes may be illustrated in Fig. 2, which presents the change of the projection onto (001) plane of the unit cell. There are two circular cylinders running through the tetragonal unit cell, and four through the hexagonal unit cell. The tetragonal cell is very similar to a pseudotetragonal unit cell found by French et al. ${ }^{\text {") }}$ for amylose-dimethylsulfoxide complex.

It seems noteworthy to compare the results of this study with that of the 8 -helix amylose described by Senti et $a l .^{2)}$ They proposed from a X-ray analysis of the fiber of tert-butyl alcohol-amylose complex containing $10 \%$ moisture that the fiber pattern is indexed on the basis of a monoclinic unit cell dimensions of $a=33.7 \AA, b=19.4 \AA, c=7.92 \AA$ (fiber axis) and $\gamma=130^{\circ}$, and two helices pass through the unit cell. The structural differences between $\gamma$ dextrin and the 8 -helix amylose complexes may be drawn from the comparison of both

9) A.D. French and H.F. Zobel, Biopolymers, 5, 457 (1967). diffraction data. Inspection of the assigned indices of the $\gamma$-dextrin- $n$-propyl alcohol complex in Table I shows that the hk0 reflection lines are missing, when $(\mathrm{h}+\mathrm{k})$ is odd, and the odd orders of h00 and $0 \mathrm{k} 0$ are also missing on the equatorial reflection lines, when $\mathrm{h}$ and $\mathrm{k}$ are odd, although $\mathrm{h} 00$ and $0 \mathrm{k} 0$ lines can not be distinguished because of the tetragonal nature of the unit cell. This fact indicates that each of the dextrin cylinders is oriented perpendicular to the (001) plane of the unit cell with its axis normal to the plane of the projection. This situation is also true of the anhydrous $\gamma$-dextrin complex which has the hexagonal unit cell. In both unit cells of $\gamma$-dextrin complexes, the projection of the cylinder onto (001) plane of the unit cell may be pictured as a circular shape as shown in Fig. 2. This situation may be quite analogous to those of $\alpha$-and $\beta$-dextrin complexes. Namely, in the preceding paper, ${ }^{11}$ it has been demon-

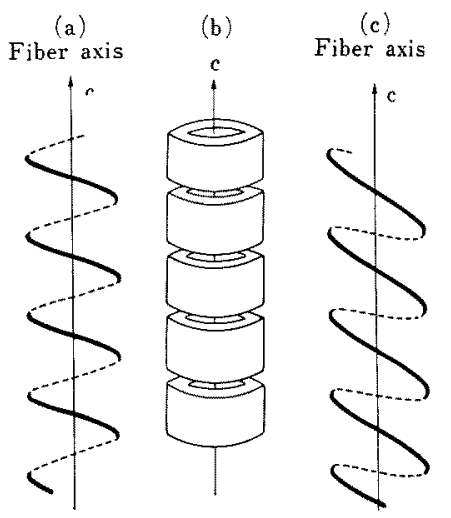

FIG. 3. Schematic Representations of the Helical Configurations of Amylose and Stacking Alignment of Cyclodextrin Molecules.

a) $61_{1}-$ and $7_{1}$-Helix amylose.

b) Cyclodextrin complexes.

c) $8_{1}$-Helix amylose.

The helical chain of amylose illustrated is lefthanded helix according to Hybl et al.10)

10) A. Hybl, R. E. Rundle and D. E. Williams J. Am. Chem. Soc., 87, 2779 (1965). 
strated that the $\alpha$ - and $\beta$-dextrin complexes with a certain kinds of organic compounds, e.g., cyclohexane, appear to have a cylindrical structure formed by coaxial stackings of the dextrin molecules, where individual planes of the dextrin molecules are oriented normal to the $\mathrm{c}$ axis as shown in Fig. 3-b. In this figure, the complexing agents enclosed as a core of the cylinder are omitted. In this case, the projection of the cylinder onto (001) plane of the unit cell may also be depicted as a circle as shown in Fig. 2-b, as was the case with $\gamma$ dextrin complexes. In addition, the dried $\alpha$ and $\beta$-dextrin complexes have been found to give identical $\mathrm{X}$-ray diffraction patterns with those of thoroughly dried $61^{-}$and $71^{-}$-helix amyloses, respectively. Accordingly, it seems likely that the projections of the $6_{1^{-}}$and $7_{1^{-}}$ helix amyloses onto (001) plane of the unit cell should also give rise to circles as shown in Fig. 2-b, indicating that the helices are circular in their cross section and can be packed together in the closest manner of circular cylinders.

On contrary to this, the unit cell of the $8_{1}$ helix amylose complex, which may well fulfil the requirements of the unit cell proposed by Senti et al., may be tentatively represented as shown in Fig. 4. It is believed ${ }^{21}$ that the $8_{1}$-helix is not circular but elliptic in its cross section as seen in Fig. 4. The only reasonable structure of the 8 -helix amylose consistent with these observations is that the individual helical loops of the $8_{1}$-helix amylose have a large inclination to a fiber axis as compared with those of the $6_{1}$ - and $7_{1}$-helix amyloses as shown in Fig. 3. Consequently, it may be said that the helical configuration of the $8_{1}$ helix amylose is quite different from those of the $61^{-}$and $7_{1}$-helix amyloses. The overall projection of such helical loops of the $8_{1}$-helix amylose onto (001) plane of the unit cell may produce the monoclinic cell proposed by Senti et al. as seen in Fig. 4 and this entirely differs from the case of $\gamma$-dextrin complexes. It appears natural that the deviation from the

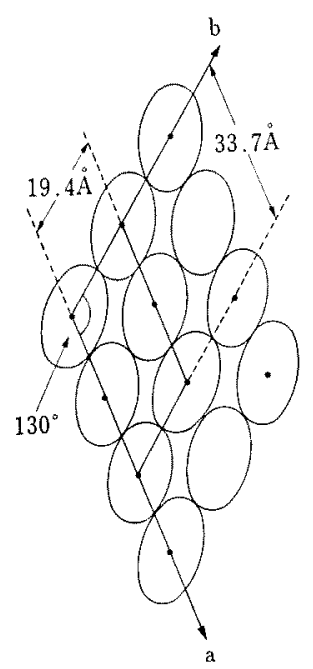

FIG. 4. Projection of the $8_{1}$-Helix Amylose onto (001) Plane According to the Suggestion of Senti et al.2)

hexagonal packing of the cylinders occurs in this cell. The reason is not clear why such a large inclination of helices to a fiber axis takes place only in the case of the $8_{1}$-helix amylose.

It was pointed out ${ }^{1 \prime}$ that the $8_{1}$-helix was never observed in thoroughly dried state in the X-ray examination of the amylose complexes with various kinds of organic compounds. On the other hand, from the studies of the destruction of amylose helices by periodate oxidation, Erlander et al. ${ }^{11 \prime}$ proposed that the helices of amylose most likely exist as an unstrained $8_{1}$-helical configuration in an aqueous solution and a powerful complexing agent such as $\mathrm{I}_{3}^{-}$is required to convert the $7_{1}$ - or $8_{1}$-helix to a strained $6{ }_{1}$-helix. In addition, Bumb et al. ${ }^{12}$ suggested from the X-ray analysis of the wet amylose complexes with halogen-substituted hydrocarbons that a diminution in the number of glucose residues per

11) S. R. Erlander, H. L. Griffin and F. R. Senti, Die Stärke, 17, 151 (1965).

12) R. R. Bumb and B. Zaslow, Carbohyd. Res., 4, 96 (1967). 
helical turn from eight to seven accompanies dehydration of amylose complex. Considering these observations, it seems likely that both the nature of the complexing agents included and water retained may play an important role in determining the size of the helices and the $8_{1}$-helix amylose might be possibl only when amylose are highly hydrated.

Acknowledgement. The authors wish express thei appreciation to Dr. T. Hattori of Kyoto Prefectura University for his kind advice in making the $\mathrm{X}$-ras analysis. 